\title{
FLORA DA SERRA DE ITABIRITO, MINAS GERAIS - PRIMEIRA CONTRIBUIÇÃO
}

\author{
Mitzi Brandão (1) \\ Manuel Losada Gavilanes (2) \\ Julio Pedro Laca-Buendia (3) \\ Lucia Helena de S. Cunha (3) \\ João Faria de Macedo (3)
}

\begin{abstract}
RESUMO - Foi efetuado o levantamento das espécies ocorrentes na Serra de Itabirito, Município de Itabirito, Minas Gerais, em áreas de cerrado e campo-rupestre. Nesta primeira etapa foram coletadas e identificadas 366 espécies, pertencentes a 209 gêneros, agrupadas em 78 famílias. Compositae é a família mais representativa, com cerca de 57 espécies e 22 gêneros, vindo a seguir Leguminosae com 37 espécies e 14 gêneros e Gramineae com 14 espécies e 13 gêneros.
\end{abstract}

Palavras-chaves: Flora de Minas Gerais, Cerrado, Campo-rupestre.

\begin{abstract}
This study is a survey of species occuring in the "cerrado" and "campo-rupreste" of Itabirito Hills, County of Itabirito, State of Minas Gerais, Brazil. In this first stage we collected and identified 366 species, belonging to 209 genera, grouped into 78 families. Compositae was the most representative family having 57 species and 22 genera, followed by Leguminosae with 37 species and 14 genera, and Gramineae with 14 species and 13 genera.
\end{abstract}

Key-words: Flora of State of Minas Gerais, "Cerrado", "Campo-rupestre”.

\section{Introdução}

Dando continuidade ao levantamento de espécies ocorrentes em áreas serranas do Estado de Minas Gerais (Brandão \& Gavilanes, 1984; Brandão et al., 1985; Ferreira \& Magalhães, 1977), enfocamos aquelas existentes na Serra de Itabirito, em município do mesmo nome (Fig. 1), no trecho cortado pela BR -

(1) Pesquisador da EPAMIG, Caixa Postal 515, 30.000 - Belo Horizonte, MG. Bolsista do CNPq.

(2) Professor de Botânica da ESAL, Caixa Postal 37, 37.200 - Lavras, MG. Bolsista do CNPq.

(3) Pesquisador da EPAMIG, caixa Postal 515, 30.000 - Belo Horizonte, MG. 
356, rodovia esta que liga Belo Horizonte a Ouro Preto.

A área escolhida apresenta as formações campestres de Cerrado e Campo Rupestre e a formação florestal: Floresta Fluvial Baixo Montana.

A referida serra apresenta inúmeras espécies afins com a Serra do Cipó, da Piedade, do Caraça, de Grão Mogol, de Ibitipoca e as Serras de Ouro Preto.

\section{Material e Métodos}

Durante 2 anos consecutivos, a área foi visitada a cada 3 meses, para coletas e/ou anotações de dados.

As coletas foram realizadas em uma área compreendida entre faixas de aproximadamente $2 \mathrm{~km}$ de largura, à direita e à esquerda da rodovia que liga Belo Horizonte a Ouro Preto, iniciando-se as mesmas, a cerca de $10 \mathrm{~km} \mathrm{da}$ maior altitude da serra, e descendo a seguir, até o sopé da mesma, no sentido Belo Horizonte - Itabirito.

O material coletado foi prensado, etiquetado, identificado e registrado no PAMG (Herbário da Empresa de Pesquisa Agropecuária do Estado de Minas Gerais - EPAMIG, Belo Horizonte - MG.).

Além do material coletado e identificado, foram examinadas exsicatas existentes nos seguintes Herbários: ESAL (Herbário do Departamento de Biologia da Escola Superior de Agricultura de Lavras, Lavras,MG.), OUPR (Herbário José Badini, da Universidade Federal de Ouro Preto, Ouro Preto, MG.), PAMG (Herbário da Empresa de Pesquisas Agropecuária de Minas Gerais, Belo Horizonte, MG.), e RB (Herbário do Jardim Botânico do Rio de Janeiro, Rio de Janeiro, RJ.).

Foram realizadas coletas nas formações Cerrado e Campo Rupestre e nas faixas de transição entre essas duas formações. A área de mata, existente na encosta próxima à cidade de Itabirito, será objeto de uma próxima etapa.

\section{Resultados e Discussōes}

Nesta Frimeira etapa, foram coletadas e identificadas, nas áreas de cerrado e campo rupestre, ocorrentes na Serra de Itabirito - no Município de Itabirito, MG., 366 espécies, pertencentes a 209 gêneros, agrupados em 78 famílias. As plantas em questão estão relacionadas nas Tabelas I e II.

Na Tabela III, as famílias estão ordenadas por ordem alfabética, seguidas do número de gêneros e de espécies encontradas. Compositae é a família mais representativa, com 57 espécies e 22 gêneros, seguida por Leguminosae (37 espécies e 14 gêneros), Melastomataceae ( 25 espécies e 13 gêneros) e Gramineae (14 espécies e 13 gêneros).

Dentre as espécies identificadas, são comuns à Serra do Caraça, segundo Ferreira et al. (1978), as espécies: Alstroemeria cunea, Anthurium sellowianum, Bilbergia amoena, Rhyncosphora globosa, Axonopus aureus, Chusquea capituliflora, Hypoxis decumbens, Pleurotalis rupestris, Laelia flava, Vellozia compacta, Ruellia macrantha, Justicia umbrosa, Staurogyne minarum, Pfaffia 
jubata, Macrosiphonia velame, Oxypetalum appendiculatum, Pyrostegia venusta, Jacaranda macrantha, Cecropia hololeuca, Achyrocline alata, Achyrocline satureoides, Baccharis aphylla, Baccharis platypoda, Eupatorium squalidum, Lychnophora ericoides, Pterocaulon alopecurioides, Trichogonia gardneri, Dasyphyllum sprengelianum, Vernonia scorpioides, Drosera graminifolia, Croton lundianus, Lippia lupulina, Sebastiana corniculata, Paliavana prasinata, Hypericum brasiliense, Camptosema scarlatinum, Periandra dulcis, Periandra coccinea, Diplusodon microphyllus, Diplusodon virgatus, Camarea ericoides, Mascagnia microphylla, Acisanthera variabilis, Lavoisiera pulcherrima, Declieuxia cordigera e Lippia lupulina.

Muitas das espécies presentes alcançam as serras de Ouro Preto (Badini, 1978; Badini \& Reis, 1969), como: Spigelia olfersiana, Macrosiphonia velame, Memora glaberrima, Adiantum cuneatum, Anemia flexuosa, Gleichenia rigida, Gleichenia furcata, Ruellia macrantha, Polygala paniculata, Cuphea ingrata, Diplusodon microphyllus, Diplusodon virgatus, Pterocaulon alopecurioides, Vernonia scorpioides, Adiantum subcordatum, Cyathea arborea, Croton lundianus, Achyrocline satureoides, Achyrocline capitata, Byrsonima variabilis, Rubus rosiifolius, Erigeron maximus, Borreria verticillata, Siphocampylus westinianus, Vanillosmopsis polycephala, Drymis brasiliensis, entre outras.

Algumas espécies vão mais além, chegando até a Serra de Grão Mogol, ao Norte do Estado (Ferreira \& Magalhães, 1977), como: Ruellia geminiflora, Baccharis gracilis, Baccharis platypoda, Drosera graminifolia, Cassia desvauxii, Camarea affinis, Camarea ericoides, Palicourea rigida, Vellozia compacta, ou ao Sul, na Serra de Ibitipoca (Ferreira \& Magalhães, 1977), a saber: Gomphrena moquini, Marcetia taxifolia, Trembleya parviflora, Microlicia isophylla, Polygala paludosa e Relbunium hipocarpium.

Muitas das espécies, coletadas na área de estudo, ocorrem ao longo da Serra do Cipó, conforme Silveira (1908), Barreto (1949), Magalhães (1954 e 1956) e Giulietti et al. (1987), a saber: Anemia elegans, Gleichenia bifida, Lycopodium cernuum, Ruellia macrantha, Alternanthera brasiliana, Pfaffia jubata, Anacardium humile, Lobelia camporum, Duguetia furfuracea, Oxypetalum appendiculatum, Begonia lobata, Jacaranda caroba, Siphocampylus macropodus, Siphocampylus westinianus, Baccharis platypoda, Baccharis trimera, Eupatorium squalidum, Mikania hirsutissima, Achyrocline alata, Achyrocline satureoides, Vernonia scorpioides, Erythroxylum campestre, Euphorbia coecorum, Camptosema scarlatinum, Stylosanthes gracilis, Stylosanthes guianensis, Stylosanthes scabra, Stylosanthes viscosa, Zornia reticulata, Cassia deusvaxii, Cassia flexuosa, Cassia rugosa, Cassia bicapsularis, Byrsonima variabilis, Camarea affinis, Camarea ericoides, Peixotoa tomentosa, Sida linifolia, Cambessedesia hilariana, Lavoisiera pulcherrima, Marcetia taxifolia, Miconia albicans, Tibouchina cardinalis, Trembleya parviflora, Ouratea floribunda, Passiflora haematostigma, Declieuxia cordigera, Lippia lupulina, Estherhazya splendida, Aegiphylla lhotzkyana, Drymis brasiliensis, Rhynchospora globosa, Axonopus aureus, Echinolaena inflexa, Mesosetum ferrugineum, Paspalum 
stellatum, Rhynchelitrum repens, Stachytarphetta glabra, Lagenocarpus rigidus, Hypoxis decumbens.

\section{Referências Bibliográficas}

BADINI, J. 1977. Roteiro para excursão botânica à Serra de Lavras Novas, Município de Ouro Preto. IN: CONGRESSO NACIONAL DE BOTÂNICA, 28, Belo Horizonte. Anais... Belo Horizonte, 1978. p. 139-143.

BADINI, J. \& REIS, M.A. 1969. Contribuição à geobotânica das Lythraceae de Minas Gerais. IN: SEMINÁRIO DE ESTUDOS FARMACÊUTICOS-BIOQUÍMICOS DE OURO PRETO, 2, Ouro Preto, 52p.

BARRETO, H.L.M. 1949. Regiōes fitogeográficas de Minas Gerais. Anuário Brasileiro de Economia Florestal, 2(2): 352-359.

BRANDĀO, M. \& GAVILANES, M.L. 1984. Subsídios para o conhecimento da vegetação da Serra da Piedade - MG. IN: CONGRESSO NACIONAL DE BOTÂNICA, 35, Manaus. Anais... (no prelo).

BRANDĀO, M.; MAGALHĀES, G.M.; GAVILANES, M.L. \& D'ASSUMPÇĀO, W.R.C. 1985. Nova contribuição para o conhecimento da vegetação da Cadeia do Espinhaço ou Serra Geral - Maciço do Caraça. IN: CONGRESSO NACIONAL DE BOTÂNICA, 36, Curitiba. Resumos, p. 214.

CRABBE, J.A.; JERMY, A.C. \& MICKEL, J.T. 1975. A new generic sequence for the pteridophyte herbarium. Fern. Gaz., 11(2-3): 141-162.

FERREIRA, M.B. \& MAGALHÃES, G.M. 1977. Contribuição para o conhecimento da vegetação da Serra do Espinhaço em Minas Gerais (Serra do Grão Mogol e da Ibitipoca). IN: CONGRESSO NACIONAL DE BOTÂNICA, 26, Rio de Janeiro, 1975. Anais... Rio de Janeiro. p. 189-202.

FERREIRA, M.B.; D'ASSUMPÇÃO, W.R.C. \& MAGALHÃES, G.M. 1978. Nova contribuição para o conhecimento da vegetação da Cadeia do Espinhaço ou Serra Geral (Maciço do Caraça). Oreádes, Belo Horizonte, 6: 46-66.

GIULIETTI, A.M.; MENEZES, N.L. de; Flora da Serra do Cipó, Minas Gerais. Caracterização e listagem das espécies. Boletim de Botânica, São Paulo, 9: 1-151.

MAGALHÃES, G.M. 1954. Contribuição para o conhecimento da Flora dos campos alpinos de Minas Gerais. IN: CONGRESSO NACIONAL DE BOTÂNICA, 5, Porto Alegre, 1954. Anais... Porto Alegre. p. 227-304.

MAGALHĀES, G.M. 1956. Características de alguns tipos florísticos de Minas Gerais. Revista Brasileira de Biologia, 1: 76-92.

SILVEIRA, A.A. 1908. Flora e Serras Mineiras. Belo Horizonte, Imprensa Oficial. 206p. 
Flora da Serra de Itabirito...

Tabela I - Relação das plantas vasculares sem sementes*, ocorrentes na Serra de Itabirito, Mun. de Itabirito-MG.

FAMÍLIA

ADIANTACEAE

ASPLENIACEAE

BLECHNACEAE

CYATHEACEAE

DAVALLIACEAE

DENNSTAEDTIACEAE

GLEICHENIACEAE

HYMENOPHYLACEAE

LYCOPODIACEAE

POLYPODIACEAE

SCHIZAEACEAE

\section{NOME CIENTÍFICO}

Adiantum capillus-veneris $\mathrm{L}$.

Adiantum cuneatum Langsd. \& Fish.

Adiantum subcordatum Swartz

Pityrogramma calomelanus (L.) Link

Pteris biaurita $\mathrm{L}$.

Pteris vittata Presl.

Asplenium auriculatum Swartz

Asplenium auritum $\mathrm{Sw}$. var. rigidum (Sw.) Hook.

Asplenium serratum $\mathrm{L}$.

Dryopteris reticulata (L.) Urb.

Blechnum glandulosum Swartz

Blechnum serrulatum Rich.

Alsophila elegans Mart.

Cyathea arborea (L.) J.E. Smith

Nephrolepis cordifolia (L.) Presl.

Lindsaea stricta (Sw.) Dryand.

Pteridum aquilinum (L.) Spr.

Gleichenia bifida (W.) Spr.

Gleichenia flexuosa (Schrad.) Mett.

Gleichenia furcata (L.) Spr.

Gleichenia longipes (Fée) Christensen

Gleichenia rigida Swartz

Hymenopyllum polyanthus Swartz

Trichomanes elegans Rich.

Lycopodium alopecurioides $\mathrm{L}$.

Lycopodium cernuum $\mathrm{L}$.

Lycopodium clavatum $\mathrm{L}$.

Lycopodium pendulinum Hook.

Polypodium angustifolum Swartz

Polypodium aureum Lowe

Polypodium pectinatum L.

Anemia ahenobarba Christensen

Anemia elegans (Gardn.) Pr.

Anemia fluxuosa (Savy) Swartz.

Anemia humilia Swatz

Lygodium polymorphum (Cav.) H.B.K.

* Segundo o Sistema de CRABBE et al. (1975) 
Tabela II - Relação das plantas vasculares com sementes*, ocorrentes na Serra de Itabirito, Mun. de Itabirito-MG.

FAMÍLIA

NOME CIENTÍFICO

FAMÍLIA

ACANTHACEAE

AMARANTHACEAE

AMARYLLIDACEAE

ANACARDIACEAE

ANNONACEAE

APOCYNACEAE

AQUIFOLIACEAE

ARACEAE

ARALIACEAE

ARISTOLOCHIACEAE

ASCLEPIADACEAE
NOME CIENTÍFICO

Justicia umbrosa (Nees) Lindl.

Ruellia geminiflora H.B.K.

Ruellia macrantha Mart.

Sericographis monticola Ness

Staurogyne minarum (Nees) Kuntze

Alternanthera brasiliana $\mathrm{L}$.

Alternanthera ficoidea $\mathrm{L}$.

Amaranthus lividus L.

Gomphrena celosioides Mart.

Gomphrena moquini Seub.

Pfaffia gnaphaloides Mart.

Pfaffia jubata Moq.

Alstroemeria cunea Vell.

Anacardium humile St.-Hil.

Schinus terebinthifolius Raddi

Annona pigmeia Mart.

Duguetia furfuracea (St.-Hil.) Benth. et Hook.

Macrosiphonia martii Muell. Arg.

Macrosiphonia velame (St.-Hil.) Muell. Arg.

Mandevilla hirsuta (A. Rich) Schum.

Mandevilla tenuifolia (Mikan) Woodson

Ilex affinis Gardn.

Anthurium sellowianum Kunth.

Didymopanax macrocarpum (Cham. \& Schl.) Seem

Didymopanax vinosum $\mathrm{E}$. March.

Aristolochia arcuata Mast.

Araujia sericifera Brotero

Ditassa ericoides Dal.

Hemipogon abietoides Fourn.

Oxypetalum appendiculatum Mart. et Zucc.

Oxypetalum erectum Mart. et Zucc.

Begonia lobata Schott.

Jacaranda caroba (Vell.) DC. 
Jacaranda macrantha Cham.

Friedericia speciosa Mart.

Memora glaberrima K. Sch.

Pyrostegia venusta Miers

BORAGINACEAE

BROMELIACEAE

CAMPANULACEAE

CECROPIACEAE

CLETHRACEAE

COMMELINACEAE

COMPOSITAE
Cordia verbenacea DC.

Heliotropium indicum L.

Aechmea aureo-rosea Baker

Bilbergia amoena (Lodd.) Lindl

Dyckia saxatilis Mez.

Tilandsia pohliana Mez.

Tilandsia usneoides $\mathrm{L}$.

Isotoma longiflora (L.) Presl.

Lobelia camporum Pohl.

Siphocampylus corymbosus Pohl.

Siphocampylus macropodus (Bilb.) G. Don

Siphocampylus nitidus Pohl.

Siphocampylus westinianus (Bilb.) Pohl.

Wahlembegia linarioides (Lam.) A. DC.

Cecropia hololeuca Miq.

Clethra brasiliensis Cham. \& Schlecht.

Commelina virginica $\mathrm{L}$.

Tradescantia elongata Meyer

Achyrocline alata DC.

Achyrocline capitata Baker.

Achyrocline satureoides (Lam.) DC.

Aspilia foliacea (Spreng.) Baker

Aspilia squarrosa Baker

Baccharis aphylla

Baccharis calvescens A.P. DC.

Baccharis dracunculifolia A.P. DC.

Baccharis genistellioides Pres.

Baccharis genistifolia A.P. DC.

Baccharis gracilis A.P. DC.

Baccharis helichryzoides A.P. DC.

Baccharis humilis Schultz.-Bip. ex Baker

Baccharis intermixa Gardn.

Baccharis itatiae Wawra

Baccharis ligustrina A.P. DC.

Baccharis lychnophora Gardn.

Baccharis myricephala A.P. DC.

Baccharis platypoda A.P. DC.

Baccharis ramosissima Gardn.

Baccharis reticulata A.P. DC.

Baccharis subdentata A.P. DC.

Baccharis trimera A.P. DC.

Baccharis varians Gardn.

Baccharis vernonioides A.P. DC.

Bidans rosiifolius DC. 
Brickelia pinifolia (Gardn.) Gray

Calea clausseniana Baker

Calea nitida Less.

Calea rotundifolia (Less.) Baker

Chaptalia integerrima (Vell.) Burk.

Dasyphyllum sprengelieanum Baker

Elephanthopus riparius Gardn.

Erigeron maximus Link. et Otto

Eupatorium laevigatum Lam.

Eupatorium squalidum DC.

Gochnata barrosii Cabr.

Lychnophora ericoides Mart.

Mikania candolleana Gardn.

Mikania cordifolia (L.f.) Willd.

Mikania hirsutissima DC.

Mikania phaeoclados Mart.

Pterocaulon alopecurioides DC.

Senecio brasiliensis Less.

Senecio vernonioides Schultz.-Bip.

Stevia lundiana DC.

Stevia urticaefolia Thumb.

Trichogonia gardneri A. Gray

Trichogonia salviaefolia Gardn.

Trixis verbascifolium Less.

Vanillosmopsis polycephala (DC.) Schultz.-Bip.

Vernonia barbata Less.

Vernonia herbacea (Vell.) Rusby

Vernonia linearifolia Less.

Vernonia scorpioides (Lam.) Pers.

Vernonia westiniana Less.

Wedelia paludosa DC.

CONVOLVULACEAE Evolvulus sp.

Ipomoea cairica (L.) Sweet.

Ipomoea longicuspis Meissn.

Ipomoea purpurea $\mathrm{Lam}$.

Ipomoea villosa Meissn.

CRUCIFERAE

CUCURBITACEAE

CYPERACEAE
Lepidium ruderale $\mathrm{L}$.

Apodanthera smilacifolia Cogn.

Echinocystis muricatus Cogn.

Bulbostylis capilaris (L.) C.B. Clabe

Bulbostylis paradoxa (Spreng.) Clark

Cyperus breviflorus L.

Cyperus ferax L.C. Rich.

Dichromena ciliata Vahl.

Dichromena setigera Kunth.

Lagenocarpus rigidus Nees

Rhynchospora consanguinea (Kunth.) Boek

Rhynchospora globosa (H.B.K.) R. \& S.

Scleria pterota Presl. 
Flora da Serra de Itabirito...

DROSERACEAE

ERICACEAE

ERIOCAULACEAE

ERYTHROXYLACEAE

EUPHORBIACEAE

GENTIANACEAE

GESNERIACEAE

GRAMINEAE

GUTTIFERAE

HIPOXIDACEAE

IRIDACEAE

LABIATAE

LEGUMINOSAE -

CAESAPINIOIDEAE
Drosera graminifolia St.-Hil.

Gaylussacia salicifolia Cham. \& Schelect.

Leucothoe pulchella DC.

Leiothrix curvifolia (Bong.) Ruhl.

Paepalanthus bryoides Kunth.

Eruthoxylum campestre St.-Hil.

Erythorxylum suberosum St.-Hil.

Croton antisyphyliticus Mart. ex Muell. Arg.

Croton lundianus Muell. Arg.

Euphorbia coecorum Boiss.

Sebastiana corniculata Muell. Arg.

Calolisianthus karmesinus Gilg.

Calolisianthus speciosus (Cham. et Sch.) Gilg.

Paliavana prasinata (Ker.) Benth.

Andropogon leucostachys H.B.K.

Aristida adscencionis $\mathrm{L}$.

Aristida pallens Cav.

Axonopus aures Beauv.

Briza neesii Doell.

Chusquea capituliflora Trin.

Ctenium cirrhosum (Ness) Kunth.

Echinolaena inflexa (Poir.) Chase

Mesosetum ferrugineum (Trin.) Chase

Panicum campestre Nees

Paspalum stellatum Humb. \& Bonpl. ex Flugge

Rhynchelitrum repens (Willd.) Hubbard.

Setaria geniculata (Lam.) Beauv.

Sporobolus poiretii (Roem. \& Schultes) Hitch.

Hypericum brasiliense Choisy

Kielmeyera corymbosa (Spreng.) Mart.

Kielmeyera variabilis Mart.

Hypoxis decumbens L.

Sisyrhinchium avenancium Klatt.

Trimezia juncifolia Benth. et Hook.

Hyptis crinita Benth.

Hytis nudicaulis Benth.

Stachys arvensis $\mathrm{L}$.

Bauhinia raddiana Berg.

Cassia bicapsularis L.

Cassia cathartica Mart.

Cassia desvauxii Collad. 
Cassia flexuosa $\mathrm{L}$.

Cassia multijuga Rich

Cassia reniformis $\mathrm{G}$. Don

Cassia repens Vog.

Cassia rotundifolia Pers.

Cassia rugosa G. Don

LEGUMINOSAE MIMOSOIDEAE

LEGUMINOSAE PAPILIONOIDEAE

LILIACEAE

LOGANICACEAE

\section{LYTHRACEAE}

Mimosa aurivilla Mart.

Mimosa vellosiana Mart. ex Benth.

Stryphnodendron barbatimao Mart.

Aeschynomene falcata (Poir.) DC.

Aeschynomene paniculata Willd.

Camptosema scarlatinum (Mart. ex Benth.) Burk.

Collaea speciosa (Lois) DC.

Crotalaria anagyroides H.B.K.

Crotalaria lanceolata E. Mey

Crotalaria nitens H.B.K.

Crotalaria rufipila Benth.

Crotalcria vespertilio DC.

Desmodium adscendens (Sw.)DC.

Desmodium incanum DC.

Desmodium uncinatum DC.

Indigofera suffruticosa Mill.

Macroptilium sp.

Periandra coccinea Benth.

Periandra dulcis Mart.

Stylosanthes gracilis H.B.K.

Stylosanthes guianensis (Aubl.) Swartz

Stylosanthes scabra Vog.

Stylosanthes viscosa Swartz

Zornia gavilanesii Brandão et Costa.

Zornia hebecarpa Mohl.

Zornia latifolia Swartz

Zornia reticulata $\mathrm{Sm}$.

Hypeastrum organense Herb.

Buddleya brasiliensis Jacq.

Spigelia olfersiana Cham. \& Schecht.

Cuphea balsamona Cham. \& Schlecht.

Cuphea carthagenensii (Jacq.) Macbr.

Cuphea ericoides Cham. et Schlecht.

Cuphea ingrata Cham. et Schlecht.

Cuphea linarioides Cham. et Schlecht.

Cuphea mesostemon Koehne

Cuphea thymoides Cham. et Schlecht.

Diplusodon buxifolius (Cham. et Schlecht.) DC.

Diplusodon calophyllum Cham. et Schlecht.

Diplusodon hirsutus DC.

Diplusodon microphyllus Pohl. 
Diplusodon replicata Pohl.

Diplusodon virgatus Pohl.

MALPIGHIACEAE

Banisteriopsis campestris (Adr. Juss.) Little

Byrsonima basiloba Adr. Juss.

Byrsonima intermedia Adr. Juss.

Byrsonima variabilis Adr. Juss.

Byrsonima verbascifolia (L.) Rich. ex A. Juss.

Camarea affinis St.-Hil.

Camarea ericoides St.-Hil.

Mascagnia microplylla Adr. Juss.) Griss.

Peixotoa tomentosa Adr. Juss.

MALVACEAE

Pavonia spinifex Cav.

Sida carpinifolia 1.

Sida cordifolia $\mathrm{L}$.

Sida glaziovii K. Schum.

Sida linifolia Cav.

Sida rhombifolia L.

Sida spinosa $\mathrm{L}$.

Urana lobata L.

\section{MELASTOMATACEAE}

Acisanthera alsinaefolia Triana

Acisanthera variabilis Triana

Cambessedesia hilariana (St.-Hil. ex Bonpl.) DC.

Cambessedesia ilicifolia Triana

Clidenia hirta (L.) G. Don

Comolia sessilis Triana

Eriocnema fulva Naud.

Lavoisiera pulcherrima Mart. \& Shr. ex DC.

Leandra sulphurea Cogn.

Marcetia taxifolia (St.-Hil. ex Bonpl.) DC.

Miconia albicans (Sw.) Triana

Miconia theaezans Cogn.

Microlicia euphorbioides Mart.

Microlicia isophyla DC.

Pyramia pityrophylla Cham.

Tibouchina adenostemon (DC.) Gogn.

Tibouchina canescens Cogn.

Tibouchina cardinalis (Bonpl.) Cogn.

Tibouchina hieracioides Cogn.

Tibouchina martialis Cogn.

Tibouchina moricandiana Baill.

Tibouchina semidecandra Cogn.

Trembleya laniflora (Don.) Cogn.

Trembleya parviflora (Don.) Cogn.

Trembleya rosmarinoides DC.

MYRSINACEAE

MYRTACEAE
Rapanea guianensis Aubl.

Campomanesia adamantinum (Camb.) Berg. Myrcia variabilis DC.

Psidium cinereum Mart. ex DC. 
NYCTAGINACEAE

OCHNACEAE

ONAGRACEAE

ORCHIDACEAE

PALMAE

PASSIFLORACEAE

PIPERACEAE

PLANTAGINACEAE

POLYGALACEAE

ROSACEAE

RUBIACEAE
Mirabilis jalapa L.

Lavradia ericoides St.-Hil.

Ouratea floribunda (St.-Hil.) Engler

Hypericum brasiliensis Choisy

Fuchsia integrifolia Camb.

Fuchsia montana Camb.

Ludwigia suffruticosa (L.) Hara

Oenothera rosea $\mathrm{L}$.

Bifrenaria thyrianthina (Lodd.) Reich. f.

Brassavola flagellaria Bard. Rodrigues

Epidendrum campestre Lindl.

Laelia cinnabarina Batem.

Laelia flava Lindl.

Oncidium flexuosum Sims

Pleurotalis rupestris Lindl.

Acrocomia aculeata (Jacq.) Lodd. et Mart.

Passiflora haematostigma Mast.

Passiflora viliosa Vell.

Piper angustifolium Ruiz et Pav.

Pothomorphe umbellata $\mathrm{L}$.

Plantago tomentosa $\mathrm{L}$.

Monnina stenophylla St.-Hil.

Polygala anguiata DC.

Polygala cuspidata DC.

Polygala longicaulis H.B.K.

Polygala papudosa Chodat.

Polygala paniculata L.

Polygala timoutou Aubl.

Polygala violacea Aubl

Rubus rosiifolis $\mathrm{Schmidt}$

Borreria verticilata (L.) G.F.W. Meyer

Coccosypselum erythocephalum Cham. \& Schlecht.

Declieuxia chiococoides Muell. Arg.

Declieuxia cordigera Mart. \& Zucc. ex Schultes

Declieuxia detoidea Muell. Arg.

Declieuxia fruticosa (Willd. ex R.\&S.) Kuntze

Declieuxia satureoides Mart. \& Zucc. ex Schult.

Relbunium hypocarpium (L.) Hemsl.

Palicourea coriacea (Cham.) Schum.

Palicourea rigida H.B.K.

Psillocarpus laricoides Mart. ex Mart. \& Zucc. 
Flora da Serra de Itabirito...

\section{SAPINDACEAE}

SCROPHULARIACEAE

SOLANACEAE

TILIACEAE

ULMACEAE

UMBELLIFERAE

VELLOZIACEAE

VERBENACEAE

VERBENACEAE

WINTERACEAE

XYRIDACEAE
Serjania lethalis St.-Hil.

Estherhazya

Scoparia dulcis $\mathrm{L}$.

Solanum americanum Mill.

Solanum lycocarpum St.-Hil.

Solanum paniculatum $\mathrm{L}$.

Luehea divaricata Mart.

Trema micrantha (L.) Blume

Eryngium pristis Cham. et Schlecht.

Vellozia compacta Mart. ex Schult. f.

Aegiphylla lhotskyana Cham.

Lantana camara L.

Lantana lilacina Desf.

Lantana trifoliata $\mathrm{L}$.

Lippia candida Cham.

Lippia florida Cham.

Lippia lupulina Cham.

Lippia marrubioides Steud.

Stachytarphetta glabra Cham.

Stachytarphetta cayanensis (L.C.Rich.) Vahl.

Drymis brasiliensis Miers.

Xyris graminosa Pohl. ex Mart. 
Tabela III - Relação das famílias, número de gêneros e número de espécies de plantas ocorrentes na Serra de Itabirito, Município de Itabirito-MG.

FAMÍLIA

ACANTHACEAE

ADIANTACEAE

AMARANTHACEAE

AMARYLLIDACEAE

ANACARDIACEAE

ANNONACEAE

APOCYNACEAE

AQUIFOLIACEAE

ARACEAE

ARALIACEAE

ARISTOLOCHIACEAE

ASCLEPIADACEAE

ASPLENIACEAE

BEGONIACEAE

BIGNONIACEAE

BLECHNACEAE

BORAGINACEAE

BROMELIACEAE

CAMPANULACEAE

CECROPIACEAE

CLETHRACEAE

COMMELINACEAE

COMPOSITAE

CONVOLVULACEAE

CRUCIFERAE

CUCURBITACEAE

CYATHEACEAE

CYPERACEAE

DAVALLIACEAE

DENNSTAEDTIACEAE

DROSERACEAE

ERICACEAE

ERIOCAULACEAE

ERYTHROXYLACEAE

EUPHORBIACEAE

GENTIANACEAE

GESNERIACEAE

GLEICHENIACEAE

GRAMINEAE

GÊNEROS ESPÉCIES

FAMÍLIA

GÊNEROS ESPÉCIES

$\begin{array}{lrr}\text { GUTTIFERAE } & 2 & 3 \\ \text { HYMENOPHYLLACEAE } & 2 & 2 \\ \text { HIPOXIDACEAE } & 1 & 1 \\ \text { IRIDACEAE } & 2 & 2 \\ \text { LABIATAE } & 2 & 3 \\ \text { LEGUMINOSAE } & 14 & 37 \\ \text { LILIACEAE } & 1 & 1 \\ \text { LOGANIACEAE } & 2 & 2 \\ \text { LYCOPODIACEAE } & 1 & 4 \\ \text { LYTHRACEAE } & 2 & 13 \\ \text { MALPIGHIACEAE } & 5 & 9 \\ \text { MALVACEAE } & 3 & 8 \\ \text { MELASTOMATACEAE } & 13 & 25 \\ \text { MYRSINACEAE } & 1 & 1 \\ \text { MYRTACEAE } & 3 & 3 \\ \text { NYCTAGINACEAE } & 1 & 1 \\ \text { OCHNACEAE } & 2 & 2 \\ \text { ONAGRACEAE } & 4 & 5 \\ \text { ORCHIDACEAE } & 6 & 7 \\ \text { PALMAE } & 1 & 1 \\ \text { PASSIFLORACEAE } & 1 & 2 \\ \text { PIPERACEAE } & 2 & 2 \\ \text { PLANTAGINACEAE } & 1 & 1 \\ \text { POLYGALACEA } & 2 & 8 \\ \text { POLYPODIACEAE } & 1 & 3 \\ \text { ROSACEAE } & 1 & 1 \\ \text { RUBIACEAE } & 6 & 11 \\ \text { RUTACEAE } & 1 & 1 \\ \text { SAPINDACEAE } & 1 & 1 \\ \text { SCHYZAEACEAE } & 2 & 5 \\ \text { SCROPHULARIACEAE } & 2 & 2 \\ \text { SOLANACEAE } & 1 & 3 \\ \text { TILIACEAE } & 1 & 1 \\ \text { ULMACEAE } & 1 & 1 \\ \text { UMBELLIFERAE } & 1 & 1 \\ \text { VELLOZIACEAE } & 1 & 1 \\ \text { VERBENACEAE } & 4 & 10 \\ \text { WINTERACCEAE } & 1 & 1 \\ \text { XYRIDACEAE } & 1 & 1\end{array}$

TOTAL - 78 famílias 
Flora da Serra de Itabirito...

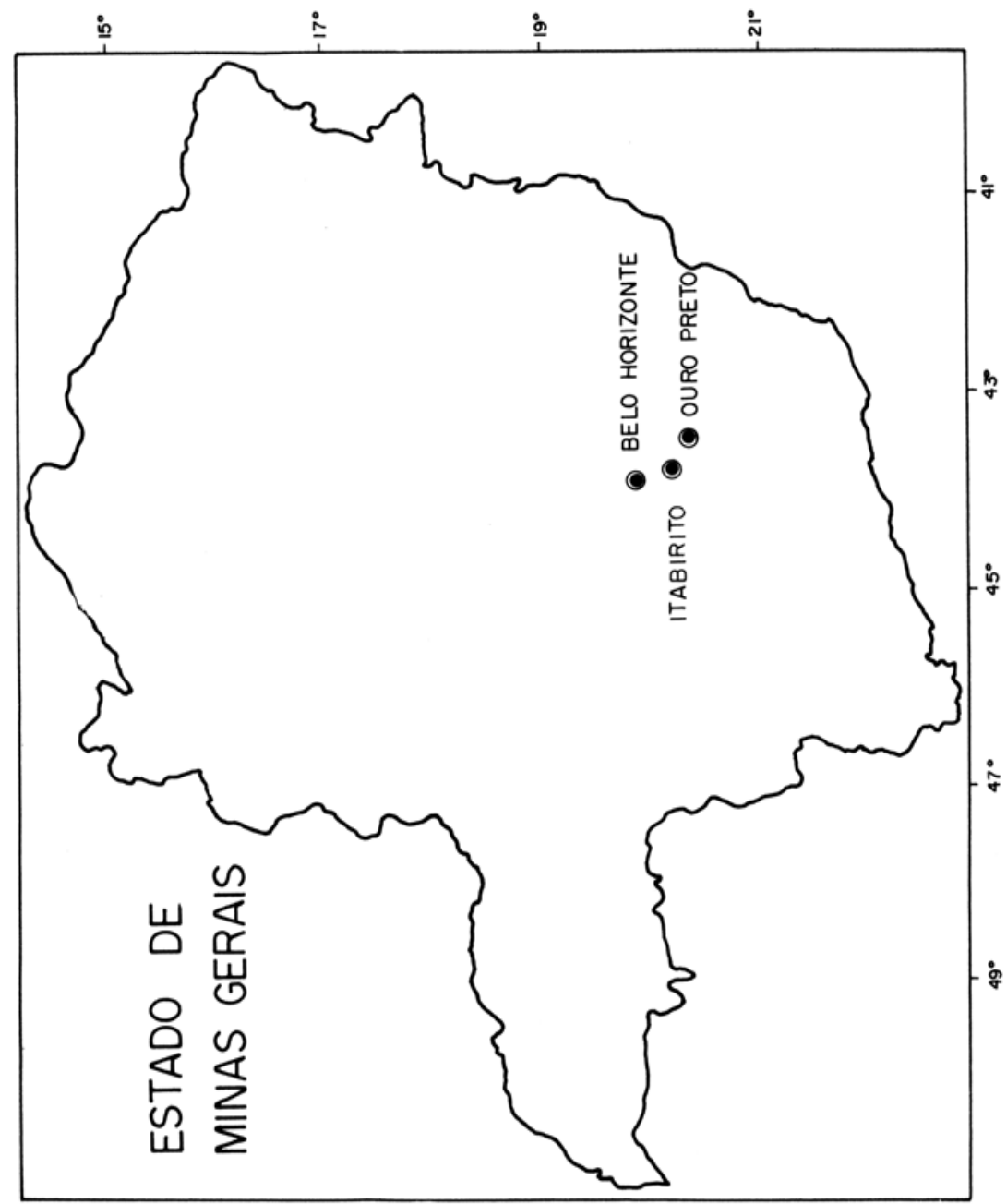

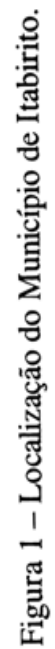

